\title{
Design and journalism - challenges and opportunities: \\ A dialogue between two cultures
}

\author{
Wibke Weber / Zurich University of Applied Sciences / Winterthur / Switzerland \\ Hans-Martin Rall / Nanyang Technological University / Singapore
}

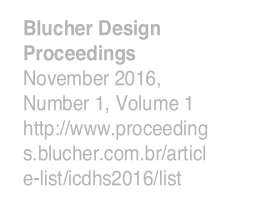

\begin{abstract}
Communication has become increasingly multi-semiotic and particularly more visual. The shift from a culture dominated by texts to a visual culture is particularly observable in journalism. Besides images, design is coming into play as a crucial semiotic mode for making meaning. In news features, special reports, or data visualizations, we can find a rich and complex interplay of different semiotic modes, e.g., text, image, and layout, which constitute the meaning-making process. However, the multimodal interplay also entails tensions since design and journalism are different disciplines with different semiotic resources, practices, and ways of thinking. In our paper, we focus on the relationship between these two disciplines by using a multimodal approach for our empirical analysis based on social semiotics. In a case study, we will examine the role of design as a semiotic mode in journalistic artifacts and discuss challenges and opportunities of the relationship betw
\end{abstract}

\section{Keywords}

Multimodal analysis, semiotic modes, design, visual journalism

\section{Introduction}

Breaking news are depicted in timelines, financial facts are reported in real time graphs, demographic data is visualized in data visualizations and infographics, revolutions and the refugee crisis are told as nonfictional comics. The way we communicate news is undergoing a radical change. Besides texts and images, visual design is coming into play as a relevant resource for making meaning. Of course, design has always been present on website and newspaper pages, particularly in form of typography and layout. What is new is that visual design is becoming an essential semiotic mode. As such design has the potential to shape the meaning of a news story and by doing so to contribute to the meaning-making process.

For a long time, journalism has been thought in terms of the verbal mode. In the last decade the media landscape has changed. Writing is no longer the central means of conveying meaning. The visual language often regarded as bystander or the inferior sister of verbal language - is becoming even more relevant and evidently discernible (cf. Küpper 2015, 2014; SND 2015). Sara Quinn, the president of the Society for News Design (SDN), quotes one of the print judges of the SDN 37 competition, " 'In the winning papers I see that every part of (the design) has meaning and is integral to the understanding of the reader,' said Joe Hutchinson, design director for Rolling Stone. Winner entries are 'not over designed. (They are) simple but effective with good use of typography with visuals. I am impressed with how much room is being devoted to visuals' ". (Quinn 2016) What has changed in journalism is that nowadays the verbal and the visual language are on a level playing field. However, it is striking that only a few scholars have started to study the role of visual design in journalism from a theoretical and practical perspective (Machin and Polzer 2015; Ananny and Crawford 2015).

In this paper we draw attention to the growing role of visual design in journalism and its semiotic resources. The aim of the paper is to show how design can shape the meaning of a story. In a case study we will examine the role of visual design as a semiotic mode adopting a multimodal approach (sections 2, 3). Following up our insights of the analysis, we will discuss implications for the collaboration between designers and journalists (section 4). The paper concludes with a summary and future perspectives (section 5). 


\section{Design as a semiotic mode}

The social semiotic approach of multimodality assumes that communication consists not only of language but always of various modes (e.g., Kress 2010, Bezemer \& Jewitt 2010). Kress defines mode as "an organizing and shaping meaning-resource" (2010: 114). Modes answer the questions: "How is the world best represented and how do I aptly represent the things I want to represent in this environment?" (Kress 2010: 116). The modes that communication experts, journalists, and designers use in their daily routine are e.g., writing, speech, image, moving image, layout, and color. Each mode refers to a set of semiotic resources. For instance, writing has words, word classes, clauses, sentences, and grammar; on a text level we have paragraphs and blocks of writing. Furthermore, writing has a set of graphical resources such as punctuation marks, font, size, or line spacing.

The semiotic resources designers and illustrators use for making meaning are e.g., colors, light, shade, lines, points, areas, size, shape, spacing, positioning, or alignment - to mention just a few (cf. Horn 1998, Bertin 1983). The resources and modes are historically and culturally shaped through social processes and practices in different social environments. They do not have a fixed meaning but meaning potentials depending on the combinations of the resources and the given context; and they have a set of affordances - the potential uses of an artifact: what can be communicated easily with the resources of the visual mode might not be expressed in the verbal mode in the same way (Kress 2010: 84-88). Writing can name things and arrange words into sentences in order to express causality, coherence, and logic, whereas images and design can provide visual evidence, can make things immediately visible and visualize complex information so that the users are able to grasp the message at a first glance.

What a mode is depends on whether a community or a culture regards it as a mode. Traditionally, journalists make use of the semiotic inventory of linguistics to reach their goal. They choose between different word classes, lexemes, and grammatical rules to produce sentences, whereas this might not qualify as a mode amongst the community of designers who are familiar with the range and potentialities of font, color, and layout (cf. Kress 2010, 84-88; van Leeuwen 2011). We argue that in the course of media convergence graphic designers and writers working together in the social environment of a newsroom must know how meaning emerges from a multimodal artifact. They must be aware of the whole inventory of both the visual and the linguistic resources. In other words, they must know, "[w]hat functions do visual elements perform best? What do words do best?" (Horn 1998: $159)$ and what is the best way to tell the story, to convey the message correctly, efficiently, and attractively.

\section{Multimodal analysis}

The multimodal approach enables us to systematically break down an artifact into its basic modes, to analyze the interplay of the modes, and to determine the set of resources available to designers. As Machin and Polzer (2015: 13) puts it, "[i]t allows us to progress from describing design decisions only in aesthetics terms." The three metafunctions of multimodality that are based on the systemic functional linguistics will serve as an analytical framework for our analysis (cf. Djonov and Knox 2014; Kress 2010).

(1) The ideational/representational metafunction communicates something about the world the world around and inside us like actions, states, or events. Regarding our case study, this leads to the research question RQ 1: What content is represented, what excluded? How is content organized semantically?

(2) The interpersonal / interactive metafunction says something about those involved in the communication - the relationship between the author(s) and the audience. This leads to the research question RQ 2: How is the content presented to the reader, factual or sensational, neutral or subjective? What attitudes are communicated?

(3) The textual / compositional metafunction refers to how the other two functions are interwoven to produce a coherent whole. This leads to the research question RQ 3: How is the page composed to make a meaningful whole from the single units?

These questions will guide us through the analysis of our case study.

\section{Case study}

For our case study, we use the special report Der Grosse Krieg - a feature on the World War I, which was published in the NZZ am Sonntag, the Sunday edition of the Swiss newspaper Neue Zürcher Zeitung. The feature extends over two spreads and contains text, a timeline, diagrams, and illustrations in a style that is reminiscent of comics (Fig. 1). We are referring to the use of black outlines ("inking") to delineate characters and environments combined with the use of flat colors. The sequential structure and the similarly spaced picture frames show further similarities to a 
traditional comic. It was awarded the European Newspaper Award 2014 (Award of Excellence) and can be seen as a representative example of the current trend in newspaper design, that is to say: visual storytelling by combining infographics, photographs, illustrations, and text (SND 2015; Küpper 2015).
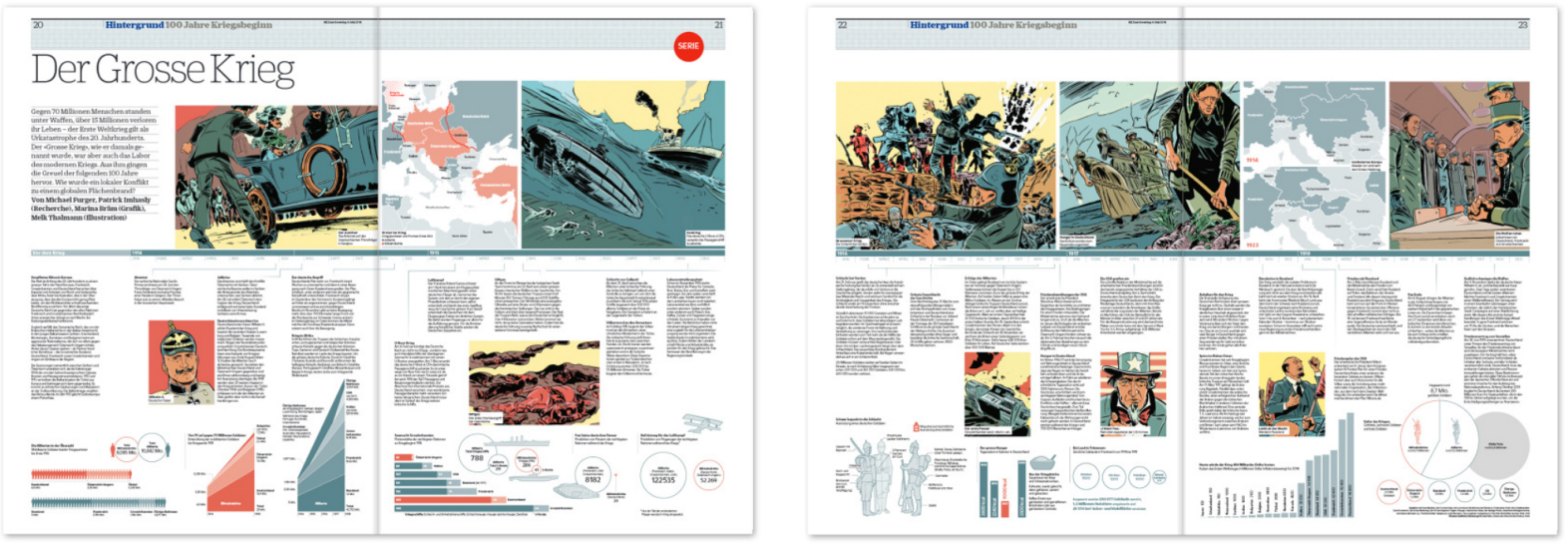

Fig. 1. Feature "Der Grosse Krieg”. NZZ am Sonntag, 4 May 2014, pp. 20-21. Production team: Michael Furger, Patrick Imhasly, Marina Bräm (infographic), Melk Thalmann (illustration). (http://marinabraem.com/DOSSIER-WORLD-WAR-1-2014)

RQ 1: What content is represented, what excluded? The topic of the special report is World War I. The social context was the centenary of the First World War in 2014. Along a timeline, the feature covers the background and origin of the war and its progress, important dates, milestones that have led to the disaster, battles, military factors, equipment, weapons, political and military alliances, economic effects, financial costs, peace treaties, the number of casualties, and aftermaths. The content is represented in three content zones: The first third of the spread depicts the story in the form of illustrations and thematic cartography (visual mode). The illustrations in comic style portray milestones of the World War I such as the trigger of the war (the assassination of Austria's Archduke Ferdinand) and the battle of Verdun. The map shows the countries that participated in the World War I. The second zone contains units of text classified with headings (mode: written language). The text units are linked to a timeline (visual-numeric mode) and illustrated by smaller pictures in the same comic style as the illustrations in the first content zone. The last third deals with the visual-numeric mode: data visualization in form of statistical graphs (cf. Weber and Engebretsen 2017). The figures of the war are depicted in bubble, pie, area, and bar charts. What attracts the reader's attention is the design of the whole page. The design does not convey the impression of a feature that deals with the First World War. It is not a text-heavy page illustrated by photographs of those years what readers might have expected - photographs are intentionally excluded. Instead, the page surprises the reader with illustrations in comic style, data visualization, and a fresh, modern and catchy look due to the color. The feature is not dominated by text. The page design follows the principle "show, don't tell".

RQ 2: How is the content presented to the reader? The communicative function of the feature is to inform and to explain, to provide facts and figures, and to give background information. This is the reason why the feature is not based on text, which is conventionally organized in columns, but resembles a big information graphic with the timeline as a backbone. The white space provides lightness and gives room to breathe and think. The font of the copy, a sans-serif typeface (which is not the usual font of the newspaper NZZ am Sonntag except headline and lead) underpins the effect of lightness and appears modest. The content zone of data visualization gives a look of objectivity and restraint; it can be read as visual arguments that corroborate the text, which is written in an objective style too. The colors used in the diagrams are soft colors, shades of blue, red, and grey, which correspond with the coloring of the illustration.

The attitude communicated in the diagrams differs strikingly from the more subjective approach of the illustrations in comic style. While layout, data visualization, and text communicate a fact-based, objective and sober attitude, the illustrations stand for emotion, sensation, drama, and disaster. The stylistic devices are typical cartooning techniques like motion lines, distorted perspectives, strong brushstrokes that provide motion, energy, and action. Here, the design mode expresses the dramatic side of the story and relates the human aspect of the story to the reader. The illustrations act as a counterbalance to the soberness of visualized data. So, the design mode represents two opposing attitudes: soberness, logical reasoning, (allegedly) objectivity because based on numbers, and 
fact-based storytelling in the data visualization versus emotion, energy, exaggeration, artistic interpretation, subjectivity, and fictional storytelling. In combination, these two aspects of the design mode present a multi-faceted view of the events, which could not be achieved in an isolated approach of either design-style: an empathetic engagement of the reader balanced with the objective facts.

RQ 3: How is the page organized? Here, the key terms are framing and salience. Visual devices that frame the feature and provide visual coherence are lines, borders, spacing, and colors. We find the same range of colors in the illustrations, the graphs, and the timeline. Lines connect the dates of the timelines to the units of text. White space and thin lines separate the different elements. At the same time, these semiotic resources provide user orientation. The layout is clearly structured, the various elements are neatly arranged however without suggesting a reading path. The reader is invited to explore the page. Only the timeline suggests a possible starting point for the reading process. The most visually salient elements that capture the reader's attraction immediately are (i) the headline and the lead and (ii) the illustrations in comic style. Especially the illustrations work as eye-catcher, which should seduce the audience to start reading the text.

Regarding typography, we find a hierarchy from the headline (in large font) to the lead (smaller font size) to the headings of the text (bold font) to the units of text (small font size, sans-serif). The pages are framed by the running title at the top and can be regarded as a corporate design element that links the feature to the wider context of the newspaper.

This short and incomplete multimodal analysis outlines exemplarily how a complex artifact can be analysed and how visual design as a semiotic mode influences its meaning. The analysis is incomplete because within the limits of this paper we cannot display the detailed analysis of images, infographics, and text.

\section{Implications for research and Practice}

The shift from a journalism ruled by text to a more visual journalism leads to complex multimodal artifacts where meaning is made not only by text but also by the images and design. This shift implies challenges and opportunities. As the production team of our case study told us, the feature was designed on a visual basis in the first place starting with the timeline and the illustrations. It is the result of a close collaboration between a graphic designer, an illustrator, and two journalists.

One challenge faced by the production was whether the comic style is appropriate to portray a severe issue such as the World War I. Comics are (still) associated with fiction. The illustrations show a subjective component since they are an interpretation of the illustrator even though they are based on original material of that time. The captions only name the events depicted in the illustrations but provide no source or link to historical documents. So, how can readers assess the historical value of the illustrations? Does the whole feature run the risk to be perceived as fictional and therefore unreliable and untrustworthy due to the comic style? How to find the right balance between fact-based storytelling, which might appear credible but boring, and fictional storytelling, which might be emotional but not authentic? Journalists strive for authenticity and factual reporting; journalism is traditionally ruled by ethical standards like accuracy in reporting, truthfulness, credibility, fairness, and objectivity. In contrast, aesthetics, creativity, originality, innovation, and entertainment belong to the toolbox of a designer rather than to a journalist's one. Design implies a subjective interpretation of the designer/illustrator in terms of color, shape, style, placement, etc. This refers in our case primarily to the illustrations, but to a lesser extent also to the design of the data visualizations even though they give us the impression of objectivity. We argue that all the ethical standards that apply to reporters also apply to designers when they are part of a journalistic team. The factual content provided by the journalistic research strongly informs the visual design process, and the visual interpretation of the designer enriches and adds to the journalistic experience. As the SND stresses in its Code of Ethics, "[l]ogic and literalness, objectivity and traditional thinking have their important place, but so must imagination and intuition, responsible creativity and empathy". (SND 2014)

Transparency is a current key term in the journalistic discourse (e.g., Silverman 2014). Transparency means that journalists disclose their opinions about certain subjects, their motives, their research methods, their sources, so that the audience can understand what actions were performed. However, in research studies in the field of journalism, visual elements including design elements have received little attention regarding transparency, credibility, and authenticity (Gynnild 2013). That leads to the next question regarding authenticity: What are possible stylistic authentication strategies visual journalists use to appear authentic and reliable? Weber and Rall (2016) have outlined several authentication strategies regarding comics journalism, e.g., providing historical photographs so that the visual journalist or illustrator reveals his or her artistic transformation from the photograph to the drawing. 
From a practical perspective the collaboration of journalists, designers, and illustrators also implicates a change in the production process and methods. Given that a multimodal artifact is designed on a visual base in the first place, sketching and storyboard become an important device for visual communication between interacting parties as well as a tool to structure the main creator's visual thought process. In general, drawing remains an ideally simple and fast tool to share ideas and discuss ideas with others (cf. Tversky and Suwa 2009). Traditionally, these visualization methods do not belong to the toolbox of journalists, however are becoming crucial in an interdisciplinary team.

These aspects mentioned above call for an interdisciplinary and transdisciplinary research approach where scholars of both disciplines are in a continuous dialogue together with practitioners. Such a dialogue, that has not started yet (Machin and Polzer 2015: 167-173), offers several opportunities. A dialogue can foster the visual literacy and critical design thinking, the awareness of design routines, the interest in analytical tools, the right handling between aesthetics and accuracy, entertainment and efficient information presentation, or to put it briefly: the mutual understanding of the vocabulary, the methods, the tools, and the way of thinking. If so, the community of journalists (whether verbal or visual) will be better positioned to design better products for the society-at-large and to master the challenges of new fields in journalism such as animated news or immersive journalism. In addition, this dialogue might contribute to the evolving field of design criticism.

\section{Concluding Remarks}

This paper has presented a multimodal approach to journalistic artifacts since written language is no longer the main mode for conveying information. Our framework can be seen as a starting point for analyzing design as a semiotic mode. Even though it is built on an approach that stems from linguistics, our framework goes beyond linguistics and accounts for the growing importance of multimodal communication, which includes design too. Further research is needed to complete the framework with categories that derive from ethnographical studies in newsrooms and interviews with designers in order to cover the full range of analysis of visual artifacts in its multiple existing incarnations, e.g., interactive visualizations (cf. Weber 2016). On a higher level, the framework can be further developed for genre and discourse analysis. Starting a dialogue between design and journalism will enable the practitioners to find effective ways of how to harness this whole inventory of semiotic resources to produce new hybrid artifacts that meet the information needs of tomorrow.

\section{References}

Ananny, M. and Crawford, K. (2015) 'A Liminal Press. Situating news app designers within a field of networked news production'. Digital Journalism, volume 3, issue 2, pp. 192-208.

Bezemer, J. and Jewitt, C. (2010) 'Multimodal analysis: Key issues', in Litosseliti, L. (ed.) Research Methods in Linguistics, London: Continuum, pp. 180-197.

Bertin, J. (1983) Semiology of Graphics: Diagrams, Networks, Maps. Madison: University of Wisconsin Press.

Djonov, E. and Knox, J.S. (2014) 'How-to-analyze webpages', in Norris, S. and Maier, C.D. (eds) Interaction, Images and Texts. A Reader in Multimodality, Boston/Berlin: De Gruyter, pp. 171-193.

Gynnild, A. (2013) 'Surveillance Videos and Visual Transparency in Journalism'. Journalism Studies, 15:4, 449-463.

Horn, R. E. (1998) Visual Language. Global Communication for the 21st Century. Bainbridge Island, WA: MacroVU.

Kress, G. (2010) Multimodality. Abingdon: Routledge.

Küpper, N. (2015) Zeitungsdesign 16: Die Ergebnisse des 16. European Newspaper Award. Meerbusch: Norbert Küpper.

Küpper, N. (2014) 'Das Besondere suchen'. viscom print \& communication, Nr. 19 (30. September 2014). 16-19.

Machin, D. and Polzer, L. (2015) Visual Journalism. London: Palgrave.

Quinn, S. (2016) 'Trends noticed by the print judges: SND37' 15 February 2016. Retrieved 23 March 2016 from http://www.snd.org/2016/02/trends-noticed-by-the-print-judges-snd37/

Silverman, C. (2014) 'The best ways for publishers to build credibility through transparency'. American Press Institute, 24 September. Retrieved 5 April 2015 from 
http://www.americanpressinstitute.org/publications/reports/strategy-studies/transparency-credibility/ SND (2015) Best of News Design. Society for News Design (ed.). Beverly, Mass.: Rockport Publishers. SND (2014) Mission/Code of Ethics. Society for News Design. Retrieved 28 January 2016 from http://www.snd.org/about/code-of-ethics/

Tversky B. and Suwa M. (2009) 'Thinking with sketches', in Markman, A (ed.) Tools for innovation. Oxford: Oxford University Press.

van Leeuwen, T. (2011) The Language of Colour: An introduction. London, New York: Routledge.

Weber, W. (2016) 'Interactive Information Graphics: A framework for classifying a visual genre', in Black, A., Lund, O. and Walker, s. (eds) The Gower Handbook of Information Design, UK: Gower, pp. 481-495. Weber, W. and Engebretsen, M. (2017) 'Graphic Modes', in Cotter, C. and Perrin, D. (eds.) Routledge Handbook of Language and Media, London, New York: Routledge (in print).

Weber, W. and Rall, H. (2016) 'Authenticity in comics journalism. Visual strategies for reporting facts.' Journal of Graphic Novels and Comics (paper accepted).

\section{Biographical note}

Prof. Dr. Wibke Weber teaches visual semiotics and digital journalism at Zurich University of Applied Sciences (ZHAW). Her research focuses on visual storytelling, genre studies, multimodal discourse analysis, and data visualization. She is editor of the book Kompendium Informationsdesign (Springer 2008) and Interaktive Infografiken (Springer 2013). Publication list: https://www.zhaw.ch/en/about-us/person/webw/

Hans-Martin Rall is an Associate Professor at Nanyang Technological University, Singapore, and an independent animation director. His films have been screened in over 250 film festivals and won more than 30 international awards. Research interest: adaptation of literature for animation. Author of the book: Animation: Konzept und Produktion (Universitätsverlag Konstanz 2015). 